\title{
Automatic Quantification of Fluorescence from Clustered Targets in Microscope Images
}

\author{
Harri Pölönen, Jussi Tohka, and Ulla Ruotsalainen \\ Tampere University of Technology, Tampere, Finland
}

\begin{abstract}
A cluster of fluorescent targets appears as overlapping spots in microscope images. By quantifying the spot intensities and locations, the properties of the fluorescent targets can be determined. Commonly this is done by reducing noise with a low-pass filter and separating the spots by fitting a Gaussian mixture model with a local optimization algorithm. However, filtering smears the overlapping spots together and lowers quantification accuracy, and the local optimization algorithms are uncapable to find the model parameters reliably. In this sudy we developed a method to quantify the overlapping spots accurately directly from the raw images with a stochastic global optimization algorithm. To evaluate the method, we created simulated noisy images with overlapping spots. The simulation results showed the developed method produced more accurate spot intensity and location estimates than the compared methods. Microscopy data of cell membrane with caveolae spots was also succesfully quantified with the developed method.
\end{abstract}

\section{Introduction}

Fluorescence microscopy is used to examine various biological structures such as cell membrane. Due to the diffraction limit, targets smaller than the optical resolution of the microscope system appear as a spot-shaped intensity distibutions in the image. A group of closely located targets with a mutual distance near the Rayleigh limit appear as a cluster of overlapping spots. The locations (with sub-pixel accuracy) and intensities of these small targets or spots are the point of interests in many applications $[1,[2,[3$. A common approach to this quantification is first to reduce the noise by filtering and then fitting Gaussian mixture model [4], [5], [6] . A low-pass filter is used in order to eliminate the high frequency noise and a Gaussian kernel is also commonly used to simplify the fitting of the mixture model to the filtered image. Another common point of interest is to estimate the number of individual spots in the image, which we will not discuss here.

When imaging small targets such as cell membrane, subtle properties and variations are to be detected, and therefore the best possible accuracy must be achieved in the image processing and analysis. Although the widely applied lowpass filter makes the image visually more appealing to the human eye due to noise reduction (see Fig. 2), valuable information is lost during filtering and the accuracy of the quantification of the spots is weakened. Also, fitting the mixture 
model to the image is not as straightforward as it is often assumed, and the fitting may introduce errors to the results if not performed properly.

In this study, we developed a procedure to to quantify the overlapping spots from the raw microscope images reliably and accurately using Gaussian mixture models and a differential evolution algorithm. We show with simulated data that this new method produces significant improvements in both the spot intensity and the location estimates. We do not filter the image, which makes the mixture model parameter estimation more challenging due to the several local optima, and we present a variant of the differential evolution algorithm that is able to determine the optimal parameters for the model.

\section{Methods}

\subsection{Model Description}

We model a raw microscope image of mutually overlapping spots with a mixture model of Gaussian components. We create an image $C_{\theta}$ according to mixture model parameters $\theta$ and determine the fitness of the parameters by mean squared error between the raw image $D$ and the created image. The value of a pixel $(i, j)$ in the image $C_{\theta}$ is defined by the probability density function of the mixture model with $k$ components multiplied by the spot intensity $\rho_{p}$ as

$$
C_{\theta}(i, j)=\sum_{p=1}^{k} \frac{\rho_{p}}{2 \pi \sqrt{|\Sigma|}} \exp \left(-\frac{1}{2}\left((i, j)-\mu_{p}\right)^{T} \Sigma^{-1}\left((i, j)-\mu_{p}\right)\right)
$$

where $\mu_{p}$ is the centroid location of the component $p$ and $\Sigma$ is the covariance matrix.

The covariance $\Sigma$ in the Equation (10) is kept fixed as in [5] and is determined according to the microscope settings as

$$
\Sigma=\left(\begin{array}{cc}
0.21 \frac{\lambda}{A} & 0 \\
0 & 0.21 \frac{\lambda}{A}
\end{array}\right),
$$

where $\lambda$ is the emission wavelength of the used fluorophore and $A$ denotes the numerical aperture of the used solvent (water, oil). It is shown in [5] that this fixed shape of the Gaussian component corresponds well to the true spot shape, i.e. the point spread function, produced by a small fluorescent target.

The location and intensity of each spot, i.e. Gaussian component in the model, are estimated together with the level of background fluorescence. The parameter set to be optimised is thereby

$$
\theta=\left(\mu_{1}, \rho_{1}, \ldots, \mu_{k}, \rho_{k}, \beta\right),
$$

where $\beta$ is the background fluorescence level. The number of components $k$ equals to the number of mutually overlapping spots and the total number of estimated parameters is $3 k+1$. 
If we denote the observed image pixel $(i, j)$ value as $D(i, j)$, the mean squared fitness function $f(\theta \mid D)$ can then be defined as

$$
f(\theta \mid D)=\frac{1}{n m} \sum_{i=1}^{n} \sum_{j=1}^{m}\left(D(i, j)-C_{\theta}(i, j)-\beta\right)^{2}
$$

where $n$ and $m$ are the image dimensions. The best parameter set $\hat{\theta}$ is then found by solving the optimization problem

$$
\hat{\theta}=\min _{\theta} f(\theta \mid D)
$$

\subsection{Modified Differential Evolution Algorithm (DE)}

Although the number of parameters in Equation (4) is not huge, it is challenging to find the parameters that minimise the squared error with high accuracy. Due to the noise in image, the parameter space is not smooth as with the filtered image but severely multimodal instead. This causes deterministic optimization algorithms to stuck easily to local optima near initial guess producing erroneous parameter estimates.

To find the optimal parameters $\hat{\theta}$, we apply a modification of differential evolution algorithm 7], which is a population-based search algorithm. Here a population member is a parameter set $\theta$ defined in Equation (3) . Unlike e.g. in genetic algorithms, in differential evolution the population is improved one member at a time and not in generation cycles. A new population candidate member $\theta_{c}$ is constructed from randomly chosen current population members $\theta_{1}, \theta_{2}$ and $\theta_{3}$ by linear combination

$$
\theta_{c}=\theta_{1}+K \cdot\left(\theta_{2}-\theta_{3}\right)
$$

where $K \in \mathbb{R}$ is a convergence control parameter. If $\theta_{c}$ has a better fit, i.e. smaller mean squared error in terms of observed image, than $\theta_{4}$, the candidate $\theta_{c}$ replaces $\theta_{4}$ in the population immediately. This procedure is repeated until all the population members are equal, and thereby the algorithm has converged.

The $K$ in Equation (6) controls the convergence rate. With high values $(K \approx$ 1.0 or above), the algorithm is very exploratory and searches the parameter space thoroughly having a good capability to finally end up near global optimum but the search may be very slow. With low values $(K \approx 0.5$ or lower) the algorithm converges faster but has a risk of converging prematurely to a local optimum. With a constant $K$ differential evolution has also a risk of stagnation [8], where the population neither evolves nor converges but rather repeats the same set of parameter values all over again.

In this study, we developed a modification of the above described algorithm to avoid the stagnation problem (see Fig. 1 for pseudo-code) and improve the performance. In our modification the convergence rate parameter $K$ from univariate distribution in the interval $[0.5,1.5]$ in each candidate $\theta_{c}$ creation by Equation (6), a new value for $K$ is randomly chosen. This guarantees that the algorithm 
will not stagnate because a different $K$ in each candidate calculation makes the candidates $\theta_{c}$ different even with the same components $\theta_{1}, \theta_{2}, \theta_{3}$ in Equation (6).

Our modification of differential evolution algorithm includes also an additional randomization step to improve the robustness of the algorithm. When the algorithm has converged and all the population members are equal, all but two population members are renewed by applying random mutations to the parameters. In practise, we multiplied each parameter of every population member with a unique random number drawn from a normal distribution with mean 1 and standard deviation 0.5. The motivation is to make the algorithm jump out of a local optimum. The algorithm is then rerun and if there is no improvement, it is assumed that the global optimum is achieved. Otherwise, if the best fit of the population was improved after the randomization, the randomization is repeated until no improvement is found. Thereby the algorithm is always run at least twice.

In our modification the population size was dependent on the number of mixture components. We used population size $30 k$, where $k$ is the number of components in the model. This is justified by the fact, that a model with more components is more complicated to estimate, and the increased population size provides more diversity to the population. We didn't include any mutation operator to the algorithm.

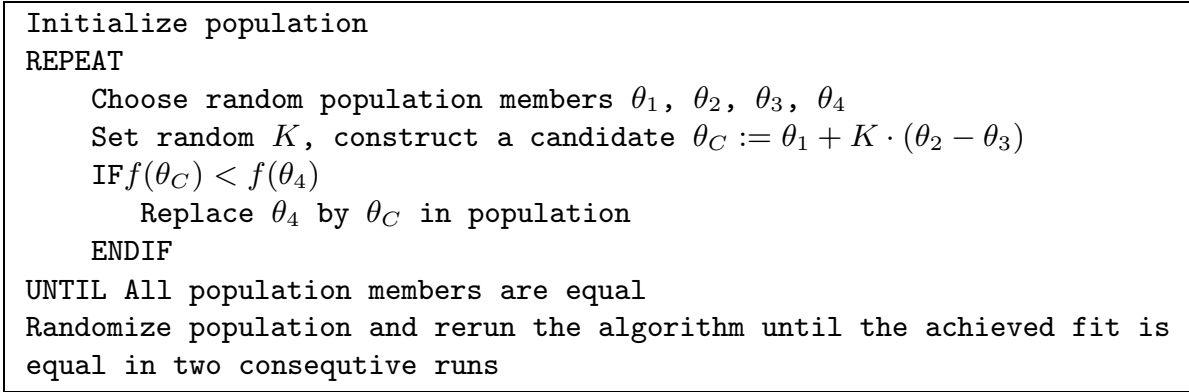

Fig. 1. Pseudo-code for modified differential evolution algorithm

\subsection{Other Methods}

As a widely used reference method to quantify the overlapping spots we use low-pass filtering and Gaussian mixture model fitting with a local non-linear deterministic algorithm. Similarly as in e.g. 5 to find the mixture model parameters we use the Levenberg-Marquardt algorithm implemented in Matlab as lsqnonlin function. We also tested the performance of the differential evolution algorithm on the filtered data, and the performance of the lsqnonlin function on the raw data. Thereby the following three methods were evaluated:

- Ref A: Filtered image and local optimization

- Ref B: Filtered image and differential evolution algorithm

- Ref C: Noisy image and local optimization 
The method A reprensents the common approach. The method B is used to test the inaccuracies produced by the local optimization algorithm in comparison to the differential evolution optimization. The method $\mathrm{C}$ is used to evaluate the effect of image filtering in Ref $\mathrm{A}$ in comparison to using the raw image. In this paper, we wanted to compare the accuracy of the methods, and therefore the correct number of components i.e. spots in each image was given to each algorithm. In practice, a spot detection method should also be implemented to determine the correct number of components.

The filter kernel in Ref A and Ref B was set as Gaussian with identity matrix as covariance matrix i.e. diagonal elements equal to one and off-diagonal elements equal to zero. In the methods $\mathrm{A}$ and $\mathrm{B}$ the fixed covariance parameter in the mixture model 1 was thereby modified to

$$
\Sigma=\left(\begin{array}{cc}
1+0.21 \frac{\lambda}{A} & 0 \\
0 & 1+0.21 \frac{\lambda}{A}
\end{array}\right)
$$

to better fit to the filtered image.

The accuracy of the deterministic optimization algorithm lsqnonlin is highly dependent on the quality of the initial guess. Here, we chose the $k$ highest local maxima in the image as an initial guess for spot centroid locations, and the sum of their surrounding eight pixels as an initial guess for spot intensity.

\section{Experimental Results}

\subsection{Simulated Data}

Simulated data was created by placing spots to overlap each other partially. The shape of a spot was determined by the theoretical point spread function defined by the Bessel function of first kind, $J_{1}$, as

$$
P(r)=\left(\frac{2 J_{1}(r a)}{r}\right)^{2} \quad \text { with } \quad a=\frac{2 \pi A}{\lambda} .
$$

Thereby, value of pixel $(i, j)$ of a spot is defined by $P(r)$, where $r$ is the distance between pixel centre to the spot centroid.

Artificial spots were located to overlap each other partially, more spesifically with a distance equal to the Rayleigh limit 9]. In cases with more than two overlapping spots, each spot had a neighbor with a distance equal to Rayleight limit, and other spots were farther away. This way, two spots never had smaller mutual distance than the Rayleigh limit and the spots were resolvable. Finally a constant background level value was added to every pixel (including pixels with spot intensity).

After creating the simulated image with point spread function spots, Poisson noise was implemented to simulate shot noise. For each pixel, we drew a random value from a Poisson distribution with parameter $\lambda$ equal to the pixel value (multiplied by a factor $\alpha$, and used this random value as the "noisy" pixel value. 

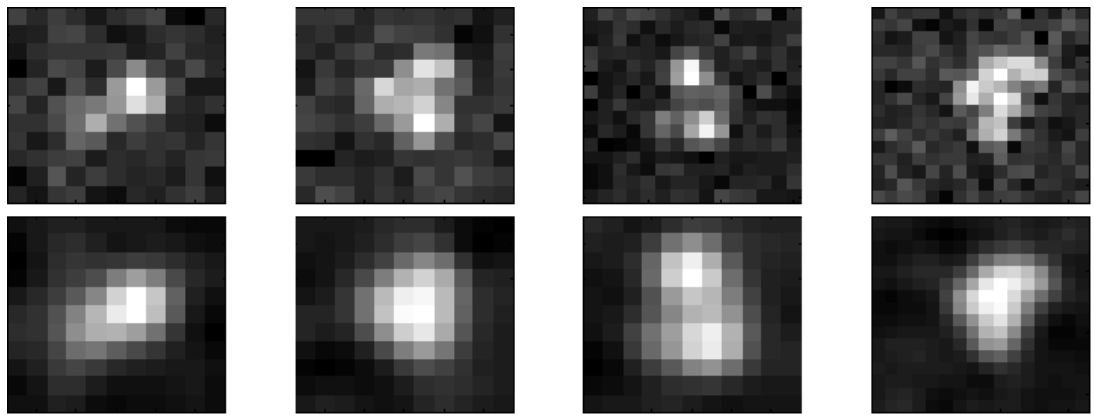

Fig. 2. Simulated data with 2 to 5 overlapping spots (left to right). Top row shows raw images with noise, bottom row shows the same images low-pass filtered.

This simulates the number of emitted photons collected by the ccd camera. With the noise multiplier $\alpha$ the signal-to-noise ratio of the images could be controlled.

In our simulated images we chose the following parameters: numerical aperture $A=1.45$, emission wavelength $\lambda=507 \mathrm{~nm}$ and image pixel size $87 \mathrm{~nm}$. These follow the setting that our collaborators have used in their biological studies. These values produced the Rayleigh limit of

$$
d=0.61 \frac{\lambda}{A}=213 n m \approx 2.45 \text { pixels },
$$

which was used as a distance between centroids of overlapping spots. Three different values were used as spot intensities: 1000, 2000 and 3000 and the background level was set to 2000 in every image. The signal-to-noise ratio was set to be 2.0 in every image by controlling the parameter $\alpha$.

Four simulated images each with a unique number of overlapping spots were created and quantified with all the methods. The easiest image had clusters of two mutually overlapping spots while the other images had three, four and in the most difficult case, five overlapping spots per cluster. There were 1000 clusters in each image. Examples of simulated overlapping spots can be seen in Figure 2

\subsection{Results with Simulated Data}

The quantification errors with simulated images can be seen in Tables 11 and 2. The spot intensity error in Table 1 is calculated as the error between the

Table 1. Median errors in spot intensities (percent)

\begin{tabular}{|c|r|r|r|r|}
\hline & \multicolumn{3}{|c|}{ METHOD } & \\
\hline Spots & Ref A & Ref B & Ref C & New \\
\hline 2 & 34.4 & 34.4 & 6.8 & 6.5 \\
\hline 3 & 32.2 & 32.2 & 7.7 & 7.0 \\
\hline 4 & 31.4 & 30.7 & 9.2 & 7.4 \\
\hline 5 & 29.5 & 28.3 & 13.5 & 8.2 \\
\hline
\end{tabular}


Table 2. Median errors in spot locations (pixels)

\begin{tabular}{|c|c|c|c|c|}
\hline & \multicolumn{3}{|c|}{ METHOD } & \\
\hline Spots & Ref A & Ref B & Ref C & New \\
\hline 2 & 0.199 & 0.199 & 0.130 & 0.124 \\
\hline 3 & 0.255 & 0.250 & 0.145 & 0.134 \\
\hline 4 & 0.304 & 0.274 & 0.176 & 0.147 \\
\hline 5 & 0.436 & 0.313 & 0.246 & 0.165 \\
\hline
\end{tabular}

estimated spot intensities and the true spot intensities in comparison to the true intensities. Perfect estimation results would produce zero percent error. The error in location in figures in Table 2 is calculated as the distance (norm) between the true spot location and the estimated spot location. Both the tables present median values within each image. Median values were used instead of mean values, because in some rare cases (less than one percent of the quantifications) deterministic optimization failed severely producing completely unrealistic results like spot intensity larger than $10^{11}$. These extreme values would affect the calculated mean error and therefore median error is more representational in this case.

As can be seen in Table1, the proposed method was the most accurate in comparison to the other methods in quantifying the spot intensities. Note that the largest error source based on these simulation results was the filtering, because the estimates obtained from filtered images (Ref A and Ref B) were significantly worse than those obtained without filtering (Ref $\mathrm{C}$ and New). This was expected because the filtering causes loss of information together with noise reduction. The improvement achieved by the stochastic optimization algorithm was especially notable with the raw data and with more complicated overlapping.

The results in estimation of spot locations in Table 2 are rather consistent with the intensity estimation results. However, it seems that the filtering increased less the error in location estimates than in intensity estimates. Nevertheless,

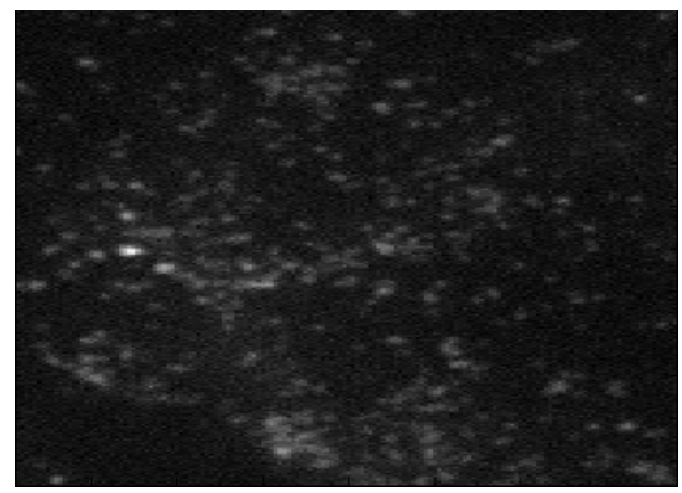

Fig. 3. A microscope image of cell membrane with caveolae 


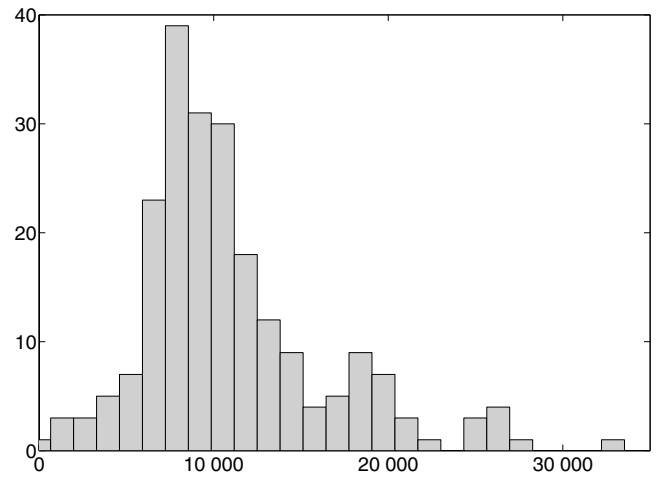

Fig. 4. Histogram of estimated intensities from a real microscope image

also in this case the new method improved the results significantly and in more complicated cases the choice of optimization algorithm seems to be crucial. The values in the Table 2 are stated in pixel units and can be converted to nanometers by multiplying with the chosen pixel size $87 \mathrm{~nm}$ to give some reference to the possible accuracy improvement with real microscopy data.

\subsection{Results with Microscopy Data}

To show that the developed method is applicable to a real microscope data, we quantify an image of a cell membrane with fluorescent caveolin-1 protein spots. The image was acquired by Institute of Biomedicine at University of Helsinki and the data has been described in detail in 10. An example of such an image can be seen in Figure 3. The intensity of spots is quantified to estimate the amount of fluorescently tagged proteins within a corresponding cell membrane invagination.

The number of individual spots within a group of overlapping spots was determined by increasing the number of components in the model iteratively until the addition didn't cause a significant improvement in the fitness of the model. Due to the fixed covariance matrix, the risk of overfitting was not so severe and the difference between significant and insignificant improvements was usually quite evident. Here, five percent improvement (or greater) was judged significant.

The results of intensity quantification with the developed method from the raw microscope image can be seen in Figure 4. There were 219 spots in total, of which 84 were overlapping with another spot. Thereby a significant portion of information would have been lost if overlapping spots have been left out of the study or quantified with poor accuracy. It can be seen in Figure 4 that the estimated intensities from clusters (at about 9000, 18000 and 27000) as expected based on biological knowledge [3], and therefore it is reasonable to assume the intensity quantification was succesful. 


\section{Conclusion}

The widely applied method to quantify fluorescence microscopy images with filtering and local optimization was found to be unoptimal for spot intensity and sub-pixel location estimation. Filtering causes significant errors especially in spot intensity estimation and reduces accuracy in the location estimation as well. Thereby the quantification should be done from the raw images, and in this study we introduced a procedure to perform such a task. The raw image quantification requires a more robust optimization algorithm and we applied a stochastic global optimization algorithm. The results with simulated data show that significant improvements were achieved in both intensity and location esimates with the developed method. Also the quantification of the microscope data of cell membrane with caveolae was succesful.

\section{Acknowledgements}

The work was financially supported by the Academy of Finland under the grant 213462 (Finnish Centre of Excellence Program (2006 - 2011)). JT received additional support from University Alliance Finland Research Cluster of Excellence STATCORE. HP received additional support from Jenny and Antti Wihuri Foundation.

\section{References}

[1] Schmidt, T., Schütz, G.J., Baumgartner, W., Gruber, H.J., Schindler, H.: Imaging of single molecule diffusion. Proceedings of the National Academy of Sciences of the United States of America 93(7), 2926-2929 (2006)

[2] Schutz, G.J., Schindler, H., Schmidt, T.: Single-molecule microscopy on model membranes reveals anomalous diffusion. Biophys. J. 73(2), 1073-1080 (1997)

[3] Pelkmans, L., Zerial, M.: Kinase-regulated quantal assemblies and kiss-and-run recycling of caveolae. Nature 436(7047), 128-133 (2005)

[4] Anderson, C., Georgiou, G., Morrison, I., Stevenson, G., Cherry, R.: Tracking of cell surface receptors by fluorescence digital imaging microscopy using a chargecoupled device camera. Low-density lipoprotein and influenza virus receptor mobility at 4 degrees c. J. Cell Sci. 101(2), 415-425 (1992)

[5] Thomann, D., Rines, D.R., Sorger, P.K., Danuser, G.: Automatic fluorescent tag detection in $3 \mathrm{D}$ with super-resolution: application to the analysis of chromosome movement. J. Microsc. 208(Pt 1), 49-64 (2002)

[6] Mashanov, G.I.I., Molloy, J.E.E.: Automatic detection of single fluorophores in live cells. Biophys. J. 92, 2199-2211 (2007)

[7] Price, K.V., Storn, R.M., Lampinen, J.A.: Differential evolution - A practical approach to global optimization. Natural computing series. Springer, Heidelberg (2007)

[8] Lampinen, J., Zelinka, I.: On stagnation of the differential evolution algorithm. In: 6th international Mendel Conference on Soft Computing, pp. 76-83 (2000)

[9] Inoue, S.: Handbook of optics. McGraw-Hill Inc., New York (1995)

[10] Jansen, M., Pietiäinen, V.M., Pölönen, H., Rasilainen, L., Koivusalo, M., Ruotsalainen, U., Jokitalo, E., Ikonen, E.: Cholesterol Substitution Increases the Structural Heterogeneity of Caveolae. J. Biol. Chem. 283, 14610-14618 (2008) 